\title{
THE ROLE OF THE STRUCTURAL CHARACTERISTIC LENGTH \\ IN FRC STRUCTURES
}

\author{
MARCO DI PRISCO ${ }^{*}$, MATTEO COLOMBO ${ }^{\dagger}$ AND ISABELLA G. COLOMBO ${ }^{\dagger \dagger}$ \\ Politecnico di Milano \\ Milano, ITALY \\ e-mail: marco.diprisco@polimi.it \\ e-mail: matteo.colombo@polimi.it \\ ${ }^{\dagger \dagger}$ e-mail: isabellagiorgia.colombo@polimi.it
}

Key words: Fiber reinforced concrete, Bending, Crack spacing, Structural characteristic length, Thin-walled elements.

\begin{abstract}
In the framework of the CEN Committee involved in the writing of the fiber reinforced concrete structure standards, a strong debate has been focused on the possibility to use a stress-strain rather than a stress- crack opening constitutive relationship, even if only the second one is physically meaningful after the cracking of the matrix. The use of a stress-strain model, even if it can be regarded as an effective simplification in many cases as it is in R/C structures, can be justified by the rough choice of a unique crack spacing in the range of $125 \mathrm{~mm}$.
\end{abstract}

In the paper, the modeling of different FRC cross sections and in particular of a thin-walled open cross-section profile longitudinally reinforced with steel bars like a FRC box-culvert (U-channel) highlights as only the use of a correct structural characteristic length when a simplified NavierBernoulli plane section model is adopted prevents the overestimation of the bearing capacity in bending. A comparison with F.E. model and previous experimental tests on full-scale structures are also proposed.

\section{INTRODUCTION}

Fiber reinforced concrete is characterised by a significant residual toughness in the postcracking regime. The simplest mechanical approach to take into account this property is the identification of a stress-crack opening constitutive law in uniaxial tension, assuming the material as a composite that can be regarded as isotropic if fibre distribution is not affected by special executing factors like wall effects or casting procedures. The pull-out contribution, that is activated only after crack opening takes place, forces the designer to introduce a structural characteristic length depending on the particular kinematic model used in the investigation. If plane section approach is used, the structural characteristic length is mainly correlated to crack spacing. If a Finite Element approach is adopted, often the rules introduced in the algorithms, calibrated for plain concrete, cannot be simply extended to FRC because they can introduce an overestimation of the dissipated energy due to the progressive increase of the crack band also 
in case of softening behaviour.

\section{REFERENCE CASES}

The choice of the structural characteristic length of a fiber reinforced concrete member is strictly related to the crack spacing of the element itself.

In this Section, some meaningful examples - useful for the definition of the crack spacing in the following Sections of the paper - are given.

Concerning bent $\mathrm{R} / \mathrm{C}$ elements with a not homogeneous reinforcement, a significant example is given by Leonhardt [1]. He showed the crack pattern of a $\mathrm{T}$ beam, in which the zone of the bottom chord strongly reinforced (4 \$26 mm) presents a small crack spacing and corresponding small crack widths; on the contrary, outside this zone, the weak web reinforcement cannot prevent wide cracks originated by larger crack spacing (Figure 1).

Another interesting example on the same subject is given by di Prisco et al. [2]. Looking at the crack pattern of a HPSFRC roof element (Figure 2), it is possible to note that the crack spacing is smaller in the prestressed chords, rather than in the flat bottom slab, which is reinforced simply with fibers (no traditional steel reinforcement) and it is mainly subjected to tensile stresses. Due to the softening behavior in uniaxial tension of the FRC used, the crack spacing in the bottom flat slab is related to the slab width (equal to about $0.83 \mathrm{~m})$.
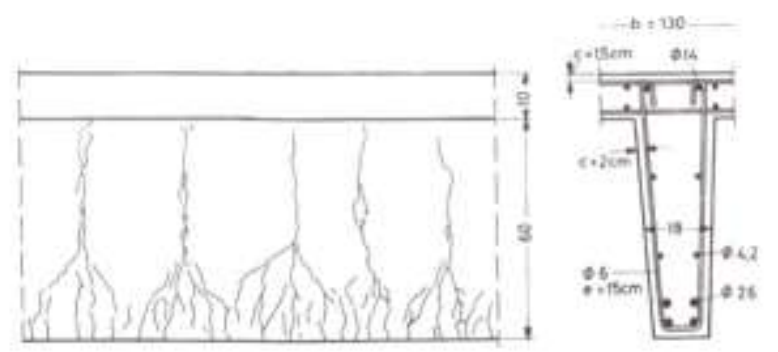

Figure 1: Crack pattern of a T beam [1].

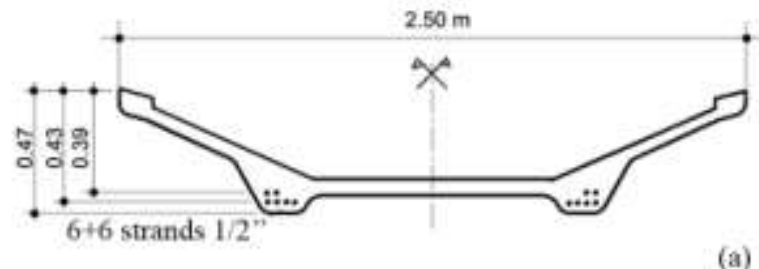

(a)

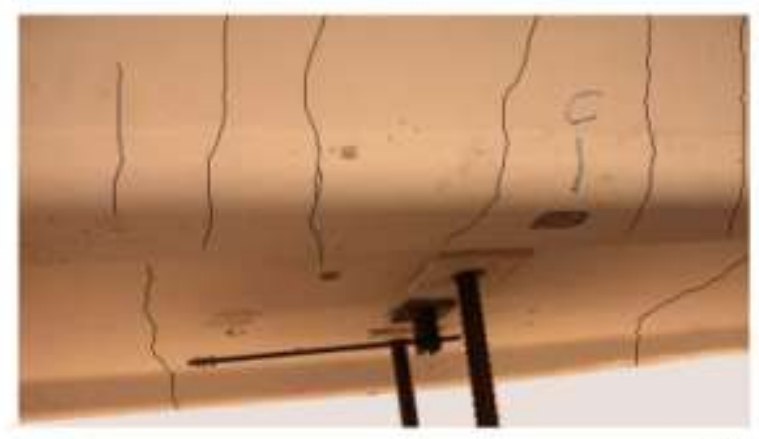

(b)

Figure 2: HPSFRC prestressed roof element: crosssection (a) and crack pattern (b) [2].

In case of deflection hardening materials and sections subjected to bending, the crack distance is related to the thickness of the bent element (Figure 3 [3]) as it occurs in plane beams subjected to a modest eccentric compression.

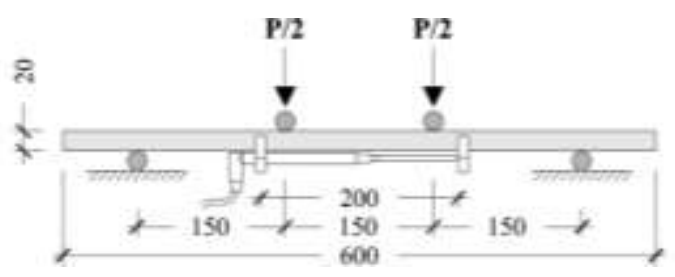

(a)

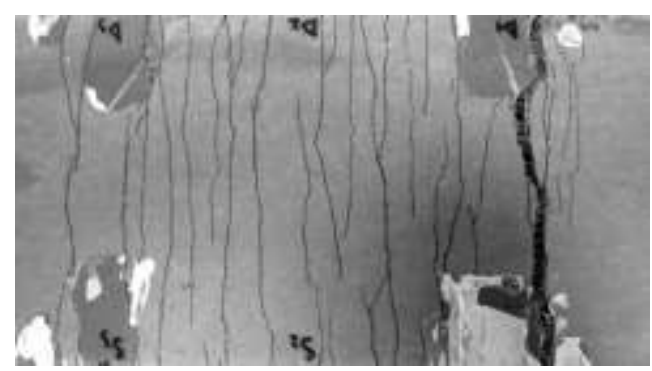

(b)

Figure 3: HPFRC plate $150 \mathrm{~mm}$ wide: four point bending test set-up (a) and crack pattern on the bottom face (b) [3]. 


\section{SHALLOW BEAM: RELIABILITY OF THE USE OF A UNIQUE CHARACTERISTIC LENGTH}

If a structural element is characterized by a section in which different structural characteristic lengths can be identified, the use of only one characteristic length in the prediction of the behavior is reliable when these characteristic lengths vary in a limited range.

To assess the truthfulness of this assertion, a fiber reinforced shallow beam cast in a prefabrication plant and tested at Politecnico di Milano is taken as a reference [4]. The beam is $1600 \mathrm{~mm}$ long and it is characterized by a rectangular cross-section $500 \mathrm{~mm}$ wide and $125 \mathrm{~mm}$ thick. It is reinforced with polypropylene fibers and a minimum steel reinforcement made of 4+4Ф6 longitudinal steel bars and $\Phi 6 / 10$ stirrups is provided (Figure 4). A four point bending test was performed on the beam considering a distance between the supports equal to $1400 \mathrm{~mm}$ and a lever arm of $500 \mathrm{~mm}$.

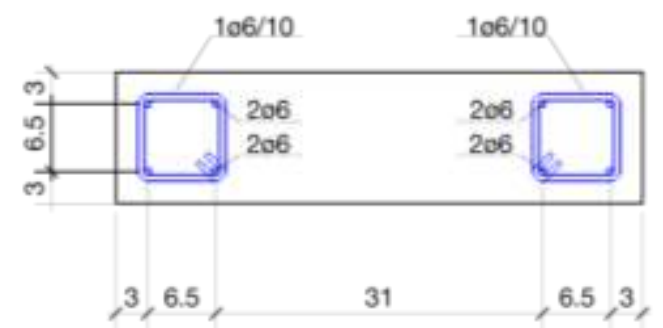

Figure 4: Shallow beam cross-section (measures in $\mathrm{cm})$.

\subsection{Materials}

The shallow beam is made of polypropylene fiber reinforced concrete. According to MC2010 [5] the concrete used to cast the beam can be classified as "C35- 2e". The properties, defined according to MC2010, are summarized in Table 1. In particular the Young's modulus $\left(E_{c i}\right)$, the characteristic compressive strength $\left(f_{c k}\right)$, the average compressive strength $\left(f_{c m}\right)$ and the lower bound value of characteristic tensile strength $\left(f_{c t k}\right)$ are collected.
Table 1: Properties of concrete class "C35"

\begin{tabular}{cccc}
\hline $\mathrm{E}_{\mathrm{ci}}[\mathrm{MPa}]$ & $\mathrm{f}_{\mathrm{ck}}[\mathrm{MPa}]$ & $\mathrm{f}_{\mathrm{cm}}[\mathrm{MPa}]$ & $\mathrm{f}_{\mathrm{ctk}}[\mathrm{MPa}]$ \\
\hline 35000 & 35 & 43 & 2.2 \\
\hline
\end{tabular}

The tensile properties of FRC were determined testing twelve specimens cured for 32 days following the procedure shown in MC2010 (Figure 5.6-5) referred to EN14651 [6]. The results are collected in Table 2 in terms of limit of proportionality $f_{L}$ and residual tensile strengths $f_{R I}$ and $f_{R 3}$, which respectively correspond to a crack mouth opening displacement $(C M O D)$ of 0.5 and $2.5 \mathrm{~mm}$. Both average and characteristic values are collected in the Table; the latter values were computed starting from average values according to the formula proposed in EN1990 [7] considering a log-normal distribution and an un-known coefficient of variation $V_{x}$.

Table 2: Tensile properties of FRC

\begin{tabular}{cccc}
\hline & $\mathrm{f}_{\mathrm{L}}[\mathrm{MPa}]$ & $\mathrm{f}_{\mathrm{R} 1}[\mathrm{MPa}]$ & $\mathrm{f}_{\mathrm{R} 3}[\mathrm{MPa}]$ \\
\hline $\mathrm{f}_{\mathrm{av}}$ & 5.05 & 3.52 & 5.54 \\
\hline $\mathrm{f}_{\mathrm{k}}$ & 4.35 & 2.14 & 3.21 \\
\hline
\end{tabular}

Yield stress $f_{y}$, ultimate strength $f_{t}$ and elongation $A_{g t}$ of steel reinforcing bars are collected in Table 3. Characteristic values were computed starting from average values according to EN1990 considering a normal distribution and an un-known coefficient of variation $V_{x}$.

Table 3: Properties of steel rebars

\begin{tabular}{cccc}
\hline & $\mathrm{f}_{\mathrm{y}}[\mathrm{MPa}]$ & $\mathrm{f}_{\mathrm{t}}[\mathrm{MPa}]$ & $\mathrm{A}_{\mathrm{gt}}[-]$ \\
\hline $\mathrm{av}$ & 559 & 590 & 0.045 \\
\hline $\mathrm{k}$ & 550 & 577 & 0.039 \\
\hline
\end{tabular}

\subsection{Constitutive laws used in the prediction}

Concerning concrete in compression, a parabolic-rectangular stress-strain relationship is adopted; the maximum strength $f_{c}$ is reached at a compressive strain equal to $2 \mathrm{e}-3$, while the failure occurs at a strain of 3.5e-3 (MC2010 Figure 7.2-8 and Equations 7.2-13 and 7.2-14).

A linear pre-cracking and linear post cracking constitutive law is used to model the FRC behavior in tension. 
According to di Prisco et al. [8], the linear post-cracking stress-CMOD behavior is identified through two points:

$\left(C M O D=0.5 \mathrm{~mm} ; \sigma=0.37 f_{R I}\right)$ and

$\left(C M O D=2.5 \mathrm{~mm} ; \sigma=0.5 f_{R 3}-k_{b} / 2 f_{R 1}\right)$,

with $k_{b}=0.529-0.143 f_{R 3} / f_{R 1}$.

According to MC2010, for bent elements the maximum value of crack opening displacement $\left(w_{u}\right)$ has to be limited to the minimum value between $2.5 \mathrm{~mm}$ and $0.02 l_{c s}$, where $1_{\mathrm{cs}}$ represents the structural characteristic length of the element.

The stress-strain relationship is obtained starting from the stress-CMOD curve by dividing the $C M O D$ by the structural characteristic length $\left(l_{c s}\right)$ of the element portion.

The characteristic length is computed as the minimum value between the mean distance between cracks $s_{r m}$ and the distance $y$ between the neutral axis and the tensile side of the cross-section (see Eq. 5.6-8, MC2010). $s_{r m}$ can be taken equal to 1.5 times the length over which slip between concrete and steel occurs (MC2010 - Equation 7.6-4, modified in 7.723).

Two cases are considered: the introduction of two characteristic lengths (one for the central part of the specimen, reinforced just with fibers, and another one for the lateral parts, reinforced with both fibers and steel bars) and the introduction of just one characteristic length for the whole section.

When two characteristic lengths are introduced, the values used are defined as follows. The central part of the specimen is loaded in bending and does not present traditional reinforcement; hence, according to MC2010, it is possible to assume $y=h=$ $125 \mathrm{~mm}$. In the lateral parts the concrete is reinforced with traditional steel rebars, hence the characteristic length is assumed equal to the lower value between $y=95 \mathrm{~mm}$ and $s_{r m}=95 \mathrm{~mm}$; hence, it is equal to $95 \mathrm{~mm}$.

When one characteristic length is introduced, it is computed considering all the section as reinforced with steel bars. Hence, it is chosen as the lower value between $y=102 \mathrm{~mm}$ and $s_{r m}=152 \mathrm{~mm}$ (which is $102 \mathrm{~mm})$.
The behavior of steel rebars is idealized through a bi-linear elasto- plastic stress-strain diagram, assuming a Young's modulus of 206 $\mathrm{GPa}$ and accounting the mechanical properties previously summarized in Table 3 .

\subsection{Comparison between experimental and analytical results}

Following a plane section approach, bending moment vs. curvature diagrams collected in Figure 5 are obtained considering both characteristic $(k)$ and mean $(m)$ material values, for both the cases in which one or two characteristic lengths are taken into account. All the analytical curves are drawn up to the point at which $w_{u}$ is reached.

In the same graph the experimental curve is plotted in order to validate the prediction. Note that the experimental curve is arrested before the peak, to preserve the LVDT transducers. The bending moment at which failure occurred is represented in the graph through a dashed line.

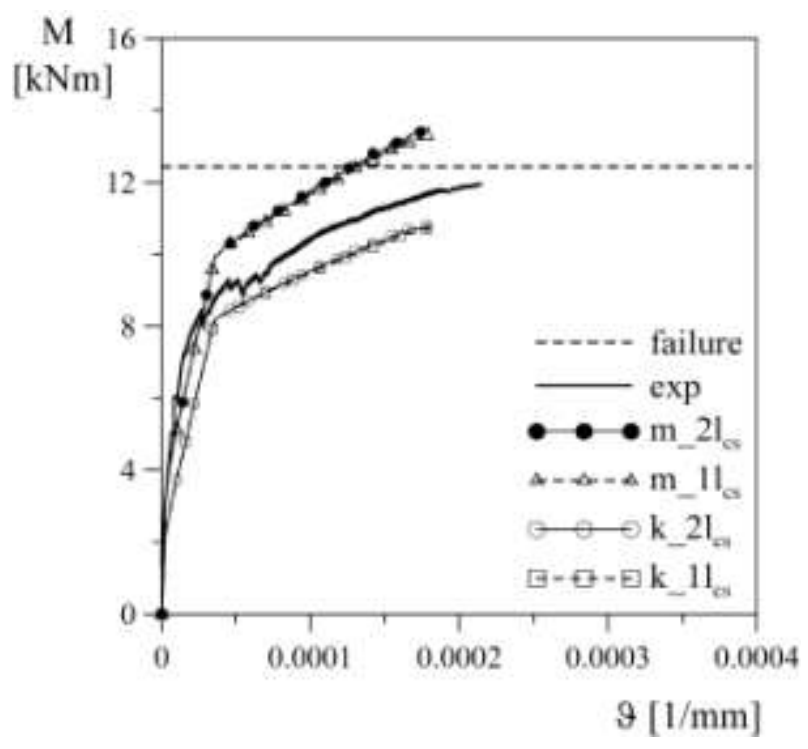

Figure 5: Shallow beam sectional behavior: bending moment vs. curvature diagrams.

It is interesting to observe how in this case the introduction of two structural characteristic lengths is fully negligible because the range of variation is limited $(95-125 \mathrm{~mm})$ and therefore a unified structural characteristic length can be adopted. 


\section{U-CHANNEL: RELIABILITY OF THE USE OF THE RIGHT STRUCTURAL CHARACTERISTIC LENGTHS}

In this Section, the modeling of a thinwalled open cross-section profile longitudinally reinforced with steel bars is proposed. The focus is placed on the different sectional responses obtained if one or more characteristic lengths are used in the model.

The geometry and the reinforcement of the culvert (U-channel) are shown in Figure 6. Proper concrete cover and bar spacing are provided in order to satisfy the MC2010 limitations. The section is reinforced providing the minimum area of steel reinforcement which allows to sustain, at the characteristic yield stress value, the load inducing the first cracking of concrete.

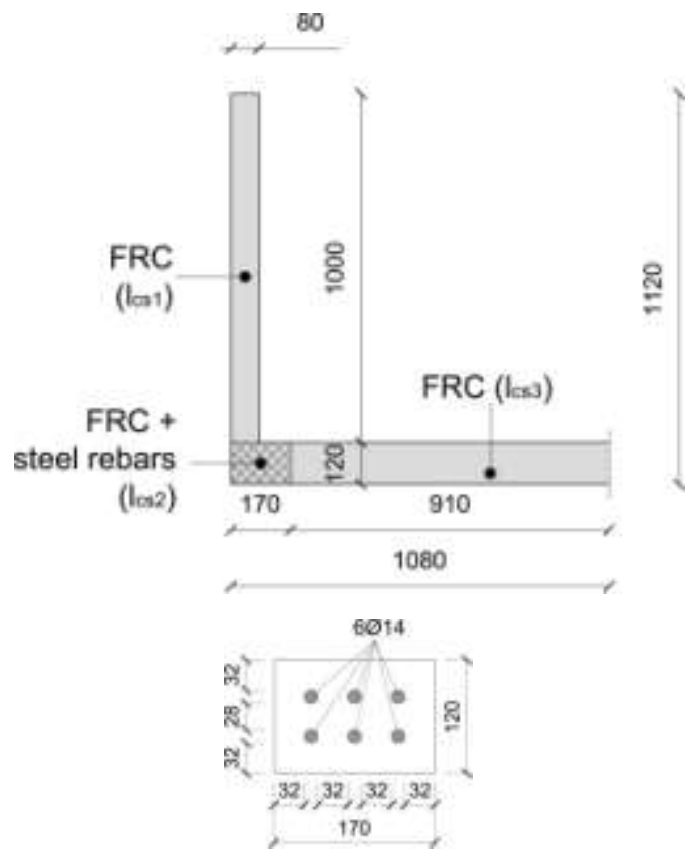

(a)

Figure 6: U-channel cross-section (measures in $\mathrm{mm}$ ): half section (a) and detail of the part reinforced with steel bars (b).

\subsection{Materials}

As in the previous example, a concrete class "C 35 " is taken into account (see Table 1).

The class of fiber reinforced concrete considered in the example is " $3 c$ " (MC2010), which is characterized by a characteristic flexural residual strength significant for serviceability conditions $\left(f_{R l k}\right)$ equal to $3 \mathrm{MPa}$ and by a characteristic flexural residual strength significant for ultimate conditions $\left(f_{R 3 k}\right)$ of $2.7 \mathrm{MPa}$.

The steel constituting reinforcing bars is characterized by a characteristic yield stress of $450 \mathrm{MPa}$ (class "B450C" in the Italian national standard NTC2008 [9]) and by a modulus of elasticity of $210 \mathrm{GPa}$.

\subsection{Constitutive laws used in the prediction}

According to MC2010 (Figure 7.2-8 and Equations 7.2-13 and 7.2-14), a parabolarectangle stress strain relationship is used for concrete in compression.

FRC in tension is modeled through two constitutive laws:

- a linear elastic - linear softening (lels) behavior;

- a bilinear hardening - bilinear softening (bh - bs) behavior.

As MC2010 classification of FRC is taken into account in this example, the constitutive laws proposed in MC2010 are used in the prediction of the channel behavior.

In the first case, the linear post-cracking stress-CMOD behavior is identified following the prescriptions proposed in Section 5.6.4 of MC2010 (linear model). In particular, it is defined through two points: $\left(0 \mathrm{~mm} ; f_{F t s}\right)$ and $\left(w_{u} ; f_{F t u}\right)$ with:

$$
\begin{gathered}
f_{F t s}=0.45 f_{R 1} \\
f_{F t u}=f_{F t s}-w_{u} / 2.5\left(f_{F t s}-0.5 f_{R 3}+0.2 f_{R 1}\right)
\end{gathered}
$$

$w_{u}$ is limited to the minimum value between $2.5 \mathrm{~mm}$ and $l_{c s} \cdot \varepsilon_{F u}$ (where $\varepsilon_{F u}$ is the ultimate strain equal to 0.02 for variable strain distribution along the cross section and to 0.01 for constant tensile strain distribution along the cross section), MC2010- Section 5.6.4. The stress-strain relationship is obtained starting from the stress-CMOD curve by dividing the $C M O D$ by the structural characteristic length, which varies according to the considered structural element portion.

In the second case, the bilinear hardening bilinear softening stress-strain relationship is built following the prescription proposed in Section 5.6.5 of MC2010 (see Figure 5.6-11a). 
In particular, the first, the second and the third branches are that of a plain concrete in uniaxial tension; the fourth branch (residual strength) is defined by two points corresponding to $\left(\varepsilon_{S L S} ; f_{F t s}\right)$ and $\left(\varepsilon_{U L S} ; f_{F t u}\right)$, with $f_{F t s}$ and $f_{F t u}$ determined as before and $\varepsilon_{S L S}$ and $\varepsilon_{U L S}$ determined as follow:

$$
\begin{gathered}
\varepsilon_{S L S}=0.5 \mathrm{~mm} / l_{c S} \\
\varepsilon_{U L S}=w_{u} / l_{c S}
\end{gathered}
$$

The maximum crack mouth opening displacement $w_{u}$ is defined as before.

For each tensile constitutive relationship, the introduction of one or three characteristic lengths is taken into account.

When one characteristic length is introduced for the whole channel section, it can be computed as the minimum value between the mean distance between cracks $s_{r m}$ and the distance $y$ between the neutral axis and the tensile side of the cross-section (bent section). Hence, $l_{c s}$ is chosen as the lower value between $y=779 \mathrm{~mm}$ and $s_{r m}=224 \mathrm{~mm}$ (which results equal to $224 \mathrm{~mm}$ ). $w_{u}$ results equal to $2.5 \mathrm{~mm}$.

When three characteristic lengths are introduced, one characteristic length is defined for the channel vertical webs, one for the slab and one for the corner chords reinforced with traditional reinforcement.

The vertical webs are loaded in bending and are characterized by the presence of steel reinforcing bars concentrated in the bottom part of each web. Hence, for each web, the characteristic length can be defined as the distance $y$ between the neutral axis and the tensile side of the L-shape bent section, which results equal to $663 \mathrm{~mm}$.

The lower slab is loaded mainly in tension and does not present any traditional reinforcement. The material is softening in uniaxial tension and therefore the crack spacing is related to the slab width [2]; hence, the characteristic length is assumed equal to $1820 \mathrm{~mm}$.

Considering the portion reinforced with traditional reinforcement, it is worth to note that this part is placed in the tensile zone of the element. The characteristic length can be assumed equal to the average spacing between cracks in a reinforced concrete member subjected to tensile load. This average distance results equal to $84 \mathrm{~mm}$.

$w_{u}$ is defined in the three cases taking into account that the webs are bent, while the slab and the $\mathrm{R} / \mathrm{C}$ parts are mainly loaded in tension. In particular, it results equal to $2.5 \mathrm{~mm}$ for the webs and the slab and to $0.84 \mathrm{~mm}$ for the reinforced $\mathrm{R} / \mathrm{C}$ portions.

As suggested by MC2010 (Figure 7.2-15), an elastic-perfectly plastic behavior is assumed for steel in tension and compression.

\subsection{Analytical results: longitudinal bending}

Following a plane section approach, bending moment vs. curvature diagrams collected in Figure 7 are obtained considering both FRC tensile constitutive laws (le - ls and bh - bs) and both the cases in which one or three characteristic lengths are taken into account.

As a reference, a curve representing the cross-sectional behavior of a reinforced concrete U-section is plotted in the graph (dashed line). In this case the tensile strength of concrete is neglected.

All the curves are obtained considering characteristic material values.

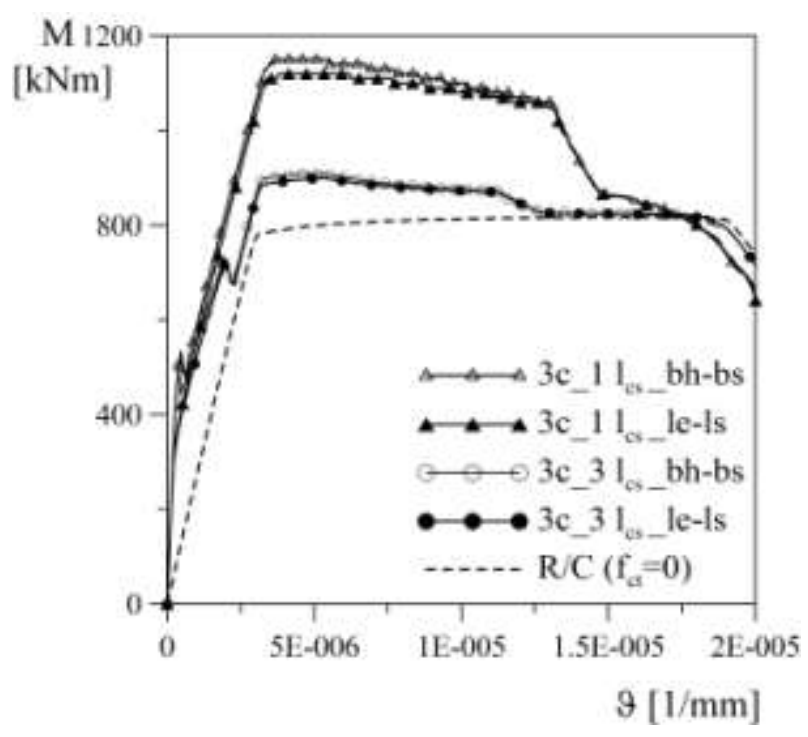

Figure 7: FRC class 3c sectional response: characteristic curves (three or one characteristic lengths; le-ls or bh-bs constitutive model) compared with the reference curve of R/C. 
Looking at the figure it is possible to observe that, in this case, the use of one or three characteristic lengths results in a completely different sectional response, showing an overestimation of the maximum bending moment in case of adopting a unique characteristic length for the whole U-shaped section. The choice of using a more accurate FRC tensile constitutive law instead of the linear model does not give rise to a significant change in the global response of the element, thus indicating that the linear model approximation is enough for predicting the mechanical behavior of the FRC structure.

In order to better understand the obtained mechanical responses, they are plotted again in Figures 8 and 9, highlighting some relevant points on the curves. In particular:

- F1 means FRC cracking at the tensile side;

- F2 means reaching of the maximum tensile strength at the tensile side when a bilinear-hardening pre-pick behavior is assumed;

- S1 means rebar yielding (subscript "inf" refers to the inferior bars, and subscript "sup" refers to the upper bars);

- C1 means reaching of the maximum compressive strength (plateau) in concrete at the compressed side;

- C2 means concrete compressive failure at the compressed side;

- $\mathrm{w}_{\mathrm{u}}$ indicates when an ultimate limit state is reached in FRC at the tensile edge $\left(\mathrm{w}_{\mathrm{u}}\right.$ refers to the case in which one characteristic length is used, while $\mathrm{w}_{\mathrm{u}_{-} \text {lcs } 1}, \mathrm{w}_{\mathrm{u} \_ \text {lcs } 2}$ and $\mathrm{w}_{\mathrm{u} \_ \text {lcs } 3}$ refer to the case in which three characteristic lengths are used and are related to the webs, the R/C portions and the slab respectively).

It is interesting to observe as in this case the reaching of the ultimate crack opening in the slab anticipates the steel yielding, due to the large value of the structural characteristic length. In the reality, the reaching of this limit does not involve a real collapse and therefore in this case the designer can renounce to the contribution of the bottom slab in tension, by continuing in the curve at least up to the reaching of $\mathrm{w}_{\mathrm{u} \_l c s 1}$ beyond which a soft softening takes place. If the designer is called to compute the ductility, the curve up to $\mathrm{C} 2$ limit can be considered.

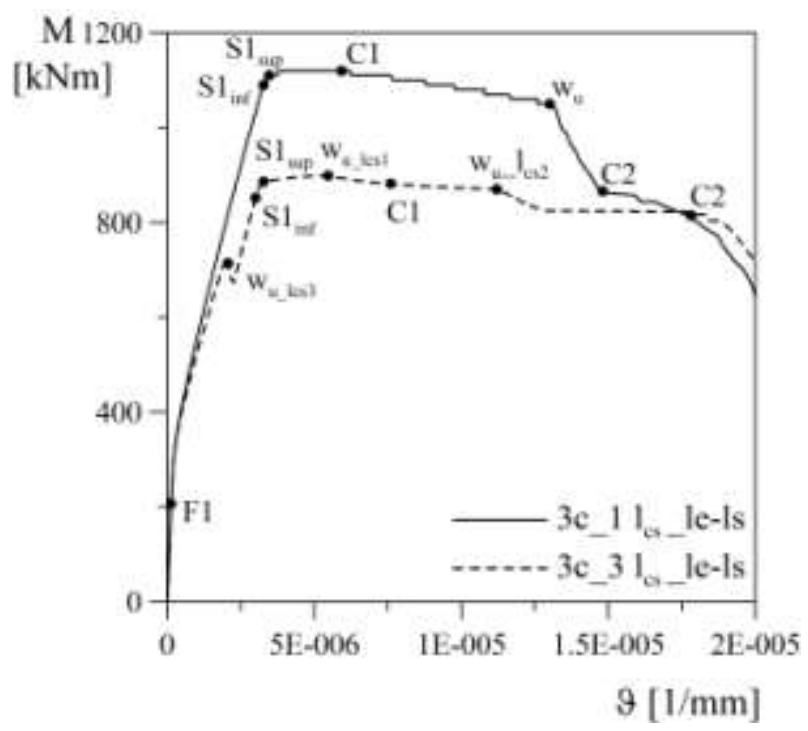

Figure 8: FRC class 3c sectional response: relevant points on characteristic curves obtained considering linear elastic - linear softening model.

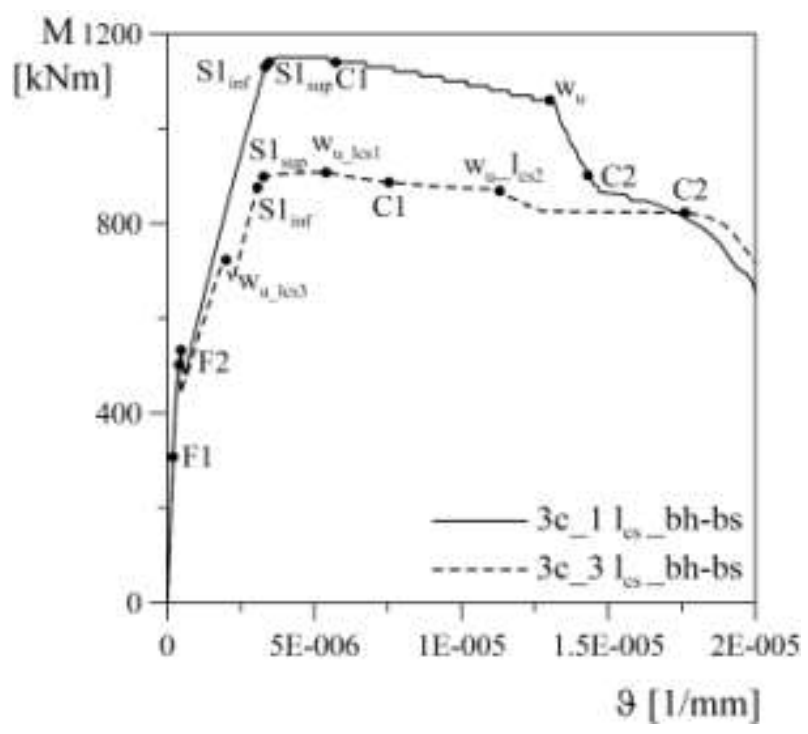

Figure 9: FRC class 3c sectional response: relevant points on characteristic curves obtained considering bi-linear hardening - bi-linear softening model.

\subsection{Analytical results: transverse bending}

As the section does not present transverse traditional reinforcement, the influence of fibers on the mechanical behavior is particularly important when transverse 
bending is investigated.

The U-channel beam is characterized by two critical bent cross-sections in transverse direction: the base web section (considering each web as a cantilever beam) and the mid slab section.

As an example, the bent web section is investigated. As the section is under bending and there is no traditional reinforcement, the characteristic length is assumed equal to the thickness of the cross-section (MC2010 Section 5.6.4).

The bending behavior is obtained assuming a width of $1 \mathrm{~m}$, hence the responses shown in Figure 10 are plotted in terms of specific moment $m$ versus curvature $\vartheta$ curves.

Also in this case characteristic curves are plotted for both FRC tensile constitutive laws (le $-1 \mathrm{~s}$ and $b h-b s$ ). The behavior of plain concrete is shown as a reference in the figure.

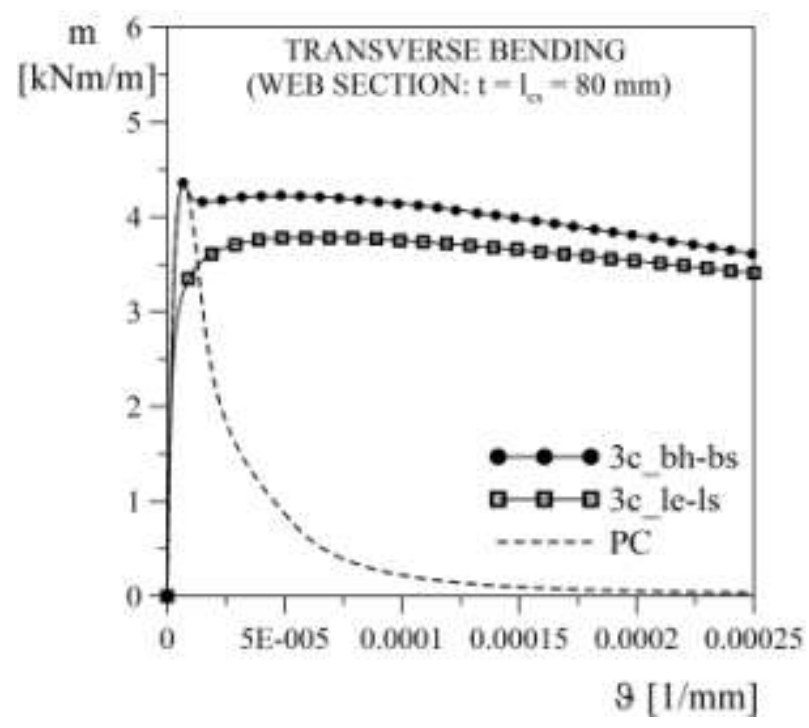

Figure 10: FRC class 3c sectional response for transverse bending: characteristic curves (le-ls or bh-bs constitutive model) compared with the reference curve of $\mathrm{R} / \mathrm{C}$.

Looking at the residual strength, the difference observed between the two curves " $3 \mathrm{c}$ le-ls" and " $3 \mathrm{c}$ bh-bs" is related to the FRC tensile relationships: the stress assumes a value equal to $f_{\text {Fts }}$ for $w=0 \mathrm{~mm}$ in the first case, and for $w=0.5 \mathrm{~mm}$ in the second case.

\section{U-CHANNEL: NUMERICAL MODEL}

A 3D numerical model has been developed in the finite element program ABAQUS Standard 6.13. The element here studied is the same investigated in Section 4. In order to obtain the sectional response preventing shear failure, a four point load test on the beam is modeled, considering a lever arm equal to $9.92 \mathrm{~m}$.

The U-shaped beam is modeled as a solid homogeneous section. Steel plates, also modeled as solid homogeneous sections, are added over the supports and under the loading knives in order to prevent any stress concentration and local failure of the element. Perfect bond is assumed between the bottom steel plates and the beam, while the top steel plates are free to move in tangential direction, in order to minimize strain concentration under the load application points.

Steel reinforcing bars are modeled through truss element embedded in the beam.

The beam and the steel plates are discretized with 8-node linear brick elements (C3D8R), and bars are discretized with 2-node linear 3-D truss elements (T3D2).

Just a quarter of the whole beam has been modeled, exploiting symmetries with respect to both $x-y$ and $y-z$ plane. The characteristics of the finite element mesh are collected in Table 4.

Table 4: FEM mesh characteristics.

\begin{tabular}{lc}
\hline Nodes (total number) & 61666 \\
\hline Elements (total number) & 43589 \\
\hline Elements type T3D2 & 1878 \\
\hline Elements type C3D8R & 41711 \\
\hline Elements on the web thickness & 2 \\
\hline Elements on the slab thickness & 3 \\
\hline Max. aspect ratio (U-shaped beam) & 1.13 \\
\hline
\end{tabular}

The model geometry with constraints is shown in Figure 11, while the mesh is shown in Figure 12. 


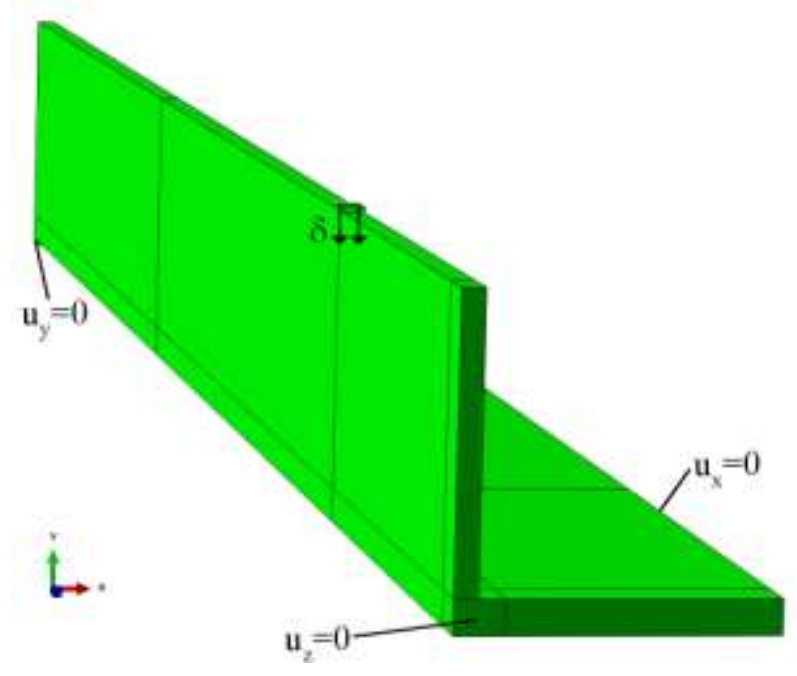

Figure 11: Finite element model: geometry with constraints.

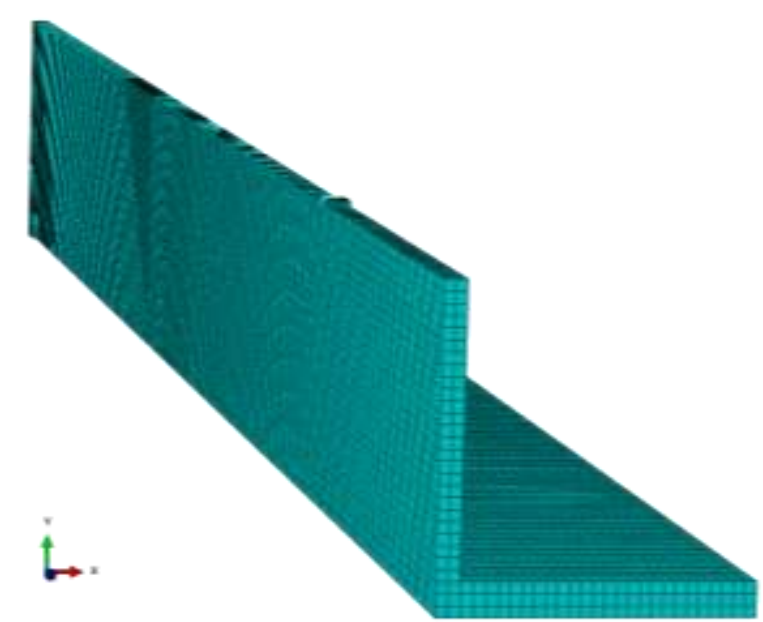

Figure 12: Finite element model: mesh.

\subsection{Constitutive laws used in the model}

The constitutive laws used in Abaqus finite element model for FRC section, steel rebars and steel plates are here summarized.

The elastic phase of fiber reinforced concrete is defined through two parameters:

- the Young's modulus, assumed equal to $35 \mathrm{GPa}$ according to Table 1;

- the Poisson's ratio, assumed equal to 0.2 .

Plasticity is introduced through Concrete Damage Plasticity model [10], which is implemented in Abaqus. Default plasticity parameters are used in the finite element analysis.

A parabolic-rectangular behavior is assumed for FRC in compression, with a maximum strength $f_{c k}$ equal to $35 \mathrm{MPa}$, reached at a strain equal to $2 \mathrm{e}-3$.

The plastic tensile behavior is defined introducing a bi-linear softening stressdisplacement relationship. The first softening branch is that of a plain concrete class "C35" characterized by a maximum tensile strength $f_{c t k}$ of $2.2 \mathrm{MPa}$. The second branch (residual strength) is defined by two points: $(0.5 \mathrm{~mm}$; $\left.f_{F t s}\right)$ and $\left(2.5 \mathrm{~mm} ; \quad 0.5 f_{R 3}-0.2 f_{R I}\right)$. No maximum crack mouth opening displacement $w_{u}$ is imposed.

The behavior of steel reinforcing bars is modeled through an elastic-perfectly plastic constitutive law, assuming a Young's modulus of $210 \mathrm{GPa}$ and a yield strength of $450 \mathrm{MPa}$.

A linear-elastic behavior is assumed for steel plates used to prevent stress concentration.

\subsection{Finite element model results}

The numerical results obtained are shown in Figure 13 in terms of bending moment (M) versus curvature $(\vartheta)$ diagram. The curvature is computed as following:

$$
\begin{gathered}
\vartheta=\left(\varepsilon_{\text {inf }}+\varepsilon_{\text {sup }}\right) / h \\
\varepsilon_{\text {inf }}=\Delta u_{z \text { zinf }} / \Delta z \\
\varepsilon_{\text {sup }}=\Delta u_{z_{\_ \text {sup }}} / \Delta z
\end{gathered}
$$

with $h$ height of the beam, $\Delta z$ width of the beam portion across the crack that localizes and $\Delta u_{z}$ elongation of the element edge. Subscript inf refers to the lower edge, while subscript sup refers to the upper edge of the beam portion.

The numerical response (dashed line) is stopped when the plastic strain of the lower edge on the beam portion considered to compute the curvature exceeds $w_{u} / l_{c s}$, computed in case of a unique characteristic length.

It is worth to note that the numerical curve is practically perfectly overlapped with the analytical response obtained using only one characteristic length. One of the reason is related to the small difference between the minimum characteristic tensile strength 
(2.2 $\mathrm{MPa})$ and the $f_{F t s}$ one $(=1.35 \mathrm{MPa})$. This small difference induces also a reduced structural characteristic length, because the ratio between the two strengths is about $60 \%$ that means that only a part of the tensile force has to be transmitted from the reinforced chords to the bottom slab.

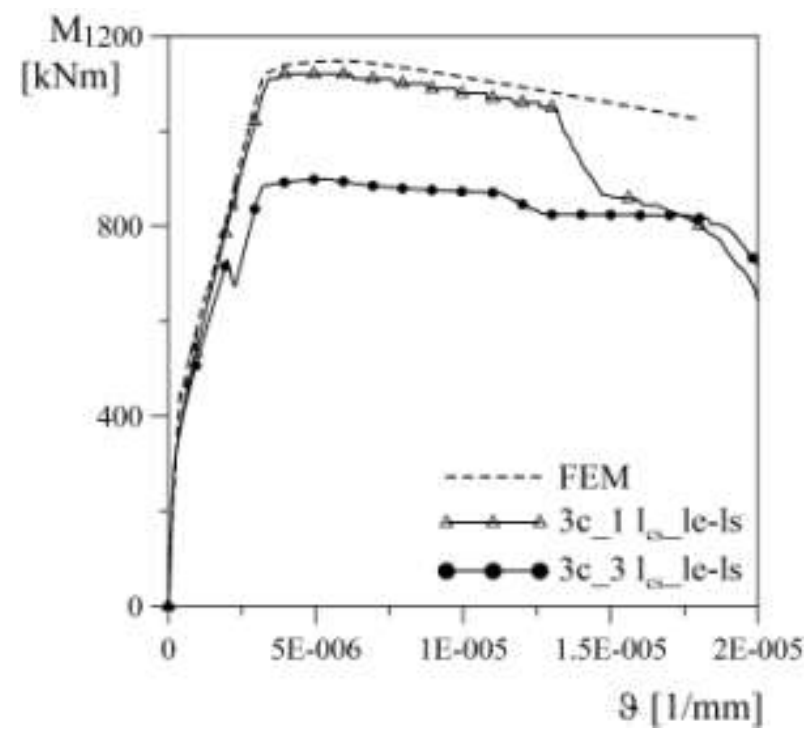

Figure 13: Finite element model results compared with analytical curves: bending moment vs. curvature diagram.

Another approximation is introduced by the formation of partial cracks, which are not at all considered in the plane-section model with different structural characteristic lengths, but that it could be often observed in real crack patterns. The crack pattern, developed according to F.E. analysis, is shown in Figure 14. It is interesting that integrating the plastic strains in the "grey" cracks a crack opening of about $2.4 \mathrm{~mm}$ can be computed, while it is only $0.4 \mathrm{~mm}$ in the "red" crack located between the previous ones. Another significant observation is that the automatic procedure introduced in the algorithm takes into account a characteristic length connected to the side length of each element, but in the macrocracks the crack band extends to five elements, thus involving a spurious energy that significantly overestimates FRC contribution in tension. This numerical effect is related to the lack of a suitable calibration of the characteristic length that is affected by the

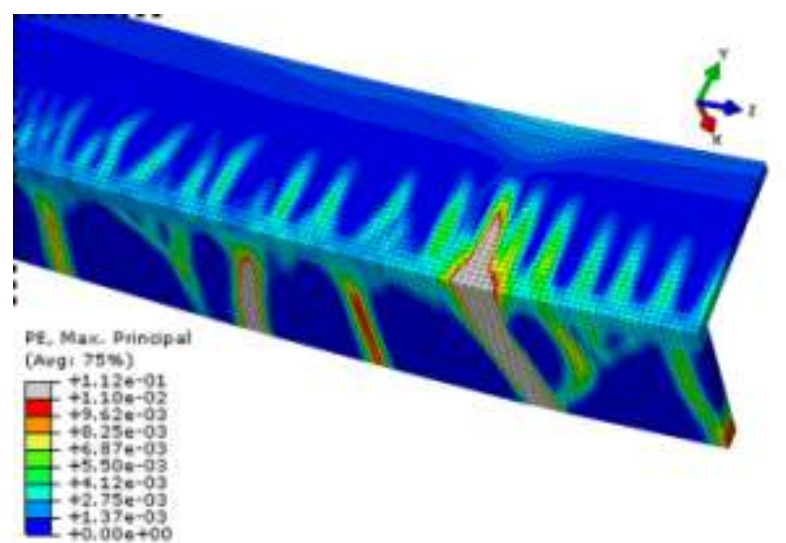

Figure 14: Finite element model results: plastic strain at the end of the numerical response.

class of the FRC investigated. It is also worth to note that in the analysis no damage was considered, thus reducing the energy release rate and thus stabilizing fictitiously the crack propagation.

\section{CONCLUSIONS}

Fiber reinforced concrete is characterized by a post-cracking residual strength that evolves with the crack opening. In order to conserve a smeared approach based on continuous strains, a structural characteristic length is required. This mechanical parameter depends on the kinematic model adopted.

Looking at a shallow beam where two cages located at the two edges scantly affect the computation of the characteristic length, because the reinforcement ratio is not homogeneous, but the variation in the width is limited, both the plane-section models assuming one or two characteristic lengths give similar predictions.

Looking at a thin-walled FRC U-channel, two extreme conditions are investigated by using a plane-section approach and then compared with a Finite Element investigation.

The results highlight a significant difference in the predicted behavior: the solution with three different lengths exhibits the lowest bearing capacity in bending (about 25\%), while Finite Element investigation gives the highest value, scantly higher than that predicted by plane-section approach, when a 
unique characteristic length is considered. The different bearing capacity is mainly affected by the correct prediction of crack spacing and a suitable calibration of the crack band width that has not to introduce a spurious energy.

\section{REFERENCES}

[1] Leonhardt, F. 1987. Cracks and crack control at concrete structures. In IABSE proceedings $\quad P$-109/87, IABSE PERIODICA 1/1987; pp. 25-44.

[2] di Prisco, M., Iorio, F., and Plizzari, G. 2003. HPSFRC prestressed roof elements. In Schnütgen, B., and Vandewalle, L. (Eds), Test and design methods for steel fibre reinforced concrete - Background and experiences PRO 31, RILEM; pp. 161-188.

[3] Zani, G. 2013. High Performance Cementitious Composites for Sustainable Roofing Panels. $\mathrm{PhD}$ thesis, Doctoral School in Structural, Seismic and Geotechnical Engineering, Politecnico di Milano.

[4] di Prisco, M., Colombo, M., Bonalumi, P., and Beltrami, C. 2014. FRC structural applications according to model code 2010: a unified approach. In Charron, J.P., Massicotte, B., Mobasher, B., and Plizzari, G. (Eds) Proceedings of the FRC 2014 Joint ACI-fib International Workshop "Fibre Reinforced Concrete: from Design to Structural Applications".

[5] fib Model Code for Concrete Structures 2010. Published by Ernst \& Sohn (2013).

[6] European standard EN 14651: 2005 - Test method for metallic fibre concrete Measuring the flexural tensile strength (limit of proportionality (LOP), residual).

[7] European standard EN 1990: 2002 Eurocode - Basis of structural design.

[8] di Prisco, M., Colombo, M., and Dozio, D. 2013. Fibre-reinforced concrete in fib Model Code 2010: Principles, models and test validation. Structural Concrete 14: 342-361.

[9] Italian standard NTC 2008 - Norme tecniche per le costruzioni.

[10] Lee, J., and Fenves, G. 1998. Plastic-
Damage Model for Cyclic Loading of Concrete Structures. Journal of Engineering Mechanics, 124(8):892-900.

[11] Ferrara, L., di Prisco, M. 2001. Mode I fracture behavior in concrete: Nonlocal damage modeling, Journal of Engineering Mechanics, 127 (7), pp. 678-692. 